\title{
A portrait of Centaur 10199 Chariklo`
}

\author{
A. Guilbert ${ }^{1}$, M. A. Barucci ${ }^{1}$, R. Brunetto ${ }^{2}$, A. Delsanti ${ }^{1}$, F. Merlin ${ }^{1,3}$, A. Alvarez-Candal $^{1}$, S. Fornasier ${ }^{1,4}$, \\ C. de Bergh ${ }^{1}$, and G. Sarid ${ }^{5}$ \\ 1 LESIA, Observatoire de Paris - Section Meudon, 2 Place J. Janssen, 92195 Meudon Principal Cedex, France \\ e-mail: aurelie.guilbert@obspm.fr \\ 2 Institut d'Astrophysique Spatiale, UMR-8617, Université Paris-Sud, bâtiment 121, 91405 Orsay Cedex, France \\ 3 Department of Astronomy, University of Maryland, College Park, MD 20742, USA \\ 4 Université Paris 7 - Denis Diderot, Paris, France \\ 5 Department of Geophysics and Planetary Sciences, Sackler Faculty of Exact Sciences, Tel Aviv University, Ramat Aviv, Israel \\ Received 14 January 2009 / Accepted 14 April 2009
}

\begin{abstract}
Context. An ESO Large Program was undertaken in October 2006 (P.I.: M. A. Barucci) to provide as complete observations as possible of about 40 Trans-Neptunian Objects and Centaurs, to investigate their surface properties. Hence, new visible and nearinfrared observations of Centaur 10199 Chariklo $\left(1997 \mathrm{CU}_{26}\right)$ ) were performed.

Aims. We investigate Chariklo's surface composition. It has already been suspected of being inhomogeneous. We try to confirm this assumption by comparing our results with previously published works, and find an explanation related the observed variations. Methods. A spectral modeling is applied to the spectra, using different types of mixtures, to place constraints on the amount of water ice present in our new spectrum. Several spectra, obtained at different moments by different groups, are compared by studying the variations in the depth of absorption bands attributable to water ice. The irradiation doses received by Chariklo's surface are also considered to interpret the observed variations.

Results. The presence of water ice is not confirmed by our featureless near-infrared spectra. The main component on the surface, identified by our spectral modeling, is amorphous carbon, which may have been produced by irradiation if Chariklo originated in the transneptunian region. The suspected surface heterogeneity is also confirmed. We show that the variations in Chariklo's spectral behaviour could be explained by a variation in the number of craters across the surface. Comet-like activity is not detected in our data, though it cannot be excluded.
\end{abstract}

Key words. Kuiper Belt - solar system: general - techniques: spectroscopic

\section{Introduction}

Centaurs are believed to be a transient population between Trans-Neptunian Objects (TNOs) and Jupiter family comets (Tiscareno \& Malhotra 2003), sharing the same physical properties as TNOs (see for example Tegler et al. 2008; Barucci et al. 2008). The Centaur object 10199 Chariklo (1997 CU26) was discovered in 1997 (Scotti 1997) by the Spacewatch Telescope. Its absolute magnitude was first determined to be $H_{V}=6.4$, corresponding to a diameter of $353 \mathrm{~km}$ (assuming a visual albedo $p_{V}$ of 0.04). Subsequent studies applied different observational techniques and derived a diameter ranging in value from 236 to $302 \mathrm{~km}$, using visual albedos from 0.04 to 0.07 (Jewitt \& Kalas 1998; Altenhoff et al. 2001; Groussin et al. 2004; Stansberry et al. 2008). Chariklo is therefore the largest known Centaur.

Photometric studies classified Chariklo in the BR class of the taxonomy relative to Trans-Neptunian Objects and Centaurs (Fulchignoni et al. 2008). From a spectroscopic point of view, Chariklo shows an interesting behaviour. Its visible spectrum is mostly featureless (Barucci et al. 1999), although Alvarez-Candal et al. (2008) found a shallow band that might be due to aqueous alteration. Its near-infrared spectrum shows evidence of variation. Water ice is indeed detected by dint of both the 1.5 and $2.0 \mu \mathrm{m}$ absorption bands, but with variable depths of between a few percent to $20 \%$ (Brown \& Koresko 1998;

* Based on observations obtained at the VLT Observatory, Cerro Paranal of European Southern Observatory, ESO, Chile, in the framework of program 178.C-0036.
Dotto et al. 2003). No feature at $1.65 \mu \mathrm{m}$ has been detected so far. Guilbert et al. (2009) presented a featureless near-infrared spectrum, with no water ice detection within the noise level. These results suggest that Chariklo's surface might be heterogeneous. However, Chariklo's rotation period is still unknown. Peixinho et al. (2001) found that it should be long (15 or $34 \mathrm{~h}$ ), and that Chariklo's absolute magnitude shows evidence of variations over a few months. Therefore, it is difficult to establish a clear relation between the observed spectra and the observed hemisphere.

Additional observations of Chariklo were carried out in the framework of the ESO Large Program begun in October 2006. The first observing run, performed in March 2007 in service mode, supplied data that were presented in part by DeMeo et al. (2009) (photometric observations), Alvarez-Candal et al. (2008) (visual spectroscopy) and Guilbert et al. (2009) $(H+K$ spectroscopy). These observations are presented in this paper together with new observations performed during a second run in visitor mode in February 2008. They are compared with several previously published results (Brown et al. 1998; Brown \& Koresko 1998; Dotto et al. 2003). Based on surface and subsurface modification processes such as irradiation and impacts, an explanation of the observed heterogeneity will also be formulated.

\section{Data observations, reduction and first analysis}

Observations of Chariklo were collected simultaneously with three instruments: FORS (visible photometry and spectroscopy), 
Table 1. Observational conditions for photometric observations.

\begin{tabular}{ccccc}
\hline \hline & Run & Filter & Exp.(sec) & Airmass \\
\hline \multirow{4}{*}{ FORS } & 20 Mar. 07 & $V$ & 80 & 1.02 \\
& 20 Mar. 07 & $R$ & 40 & 1.02 \\
\cline { 2 - 5 } & 03 Feb. 08 & $V$ & 30 & 1.14 \\
& 03 Feb. 08 & $R$ & 30 & 1.14 \\
& 03 Feb. 08 & $I$ & 45 & 1.14 \\
\hline \multirow{7}{*}{ ISAAC } & 20 Mar. 07 & $J$ & 90 & 1.25 \\
& 20 Mar. 07 & $H$ & 45 & 1.23 \\
& 20 Mar. 07 & $K$ & 30 & 1.22 \\
\cline { 2 - 5 } & 03 Feb. 08 & $J$ & 180 & 1.14 \\
& 03 Feb. 08 & $H$ & 60 & 1.13 \\
& 03 Feb. 08 & $K$ & 70 & 1.12 \\
\hline
\end{tabular}

ISAAC ( $J, H, K$ photometry and $J$ spectroscopy) and SINFONI $(H+K$ spectroscopy). Since the SINFONI exposure times were the longest, the simultaneity was only achievable if FORS and ISAAC observations were performed during the SINFONI observation, which was the case for the two observational runs.

\subsection{Photometric data}

Visible data were taken with FORS1 (FOcal Reducer and low dispersion Spectrograph), mounted on ESO-VLT UT2. The instrument is equipped with a Tek Backside thinned CCD, with $2048 \times 2048$ pixels, covering a $6.8^{\prime} \times 6.8^{\prime}$ field of view. The first run (March 2007) was performed in service mode, and photometry was taken for only two colors (Bessel $V$ and $R$ filters). FORS1's CCD was upgraded in April 2007 with two $2000 \times$ 4000 E2V CCDs, optimized in the blue range $(\lambda<0.6 \mu \mathrm{m})$. Strong fringing was observed for $\lambda>0.65 \mu \mathrm{m}$, corresponding to the $I$ Bessel filter, as well as a decrease in the spectra signal-tonoise ratio detected in this region. For this reason, we decided to use FORS2, mounted on UT1, for the second run (February 2008 ) in visitor mode. This instrument was used with the same instrumental settings previously discussed. $V, R$, and $I$ Bessel filters were used for photometry. For both runs, data reduction was performed using standard Midas and IRAF procedures (see Fornasier et al. 2004). Instrumental magnitudes were extracted in an aperture of size equal to three times the average seeing. Landolt fields were used for the calibration.

Near-Infrared photometry was performed with ISAAC (Infrared Spectrometer and Array Camera), mounted on ESOVLT UT1. The instrument is equipped with a $1024 \times 1024$ pixels Hawaii Rockwell array, which provides a $2.5^{\prime} \times 2.5^{\prime}$ field of view. We used the short wavelength arm, and $J, H, K$ s filters centered at $1.25,1.65$, and $2.16 \mu \mathrm{m}$ respectively. We used the "auto-jitter" mode to dither the telescope according to a random pattern (within 15" of the central position) in-between the different sub-exposures of an image. With this standard pointing technique, we were able to estimate the background sky contribution within each frame of a cube and apply a correction. Data reduction was performed with the ISAAC reduction pipeline using Eclipse packages. Flux were extracted using an aperturecorrection method. The calibration of magnitudes was completed using the UKIRT (United Kingdom InfraRed Telescope) faint standard stars.

The different observational conditions can be found in Table 1: for the two observing runs we summarize the different filters used, the exposure time, and the airmass at which the observations was obtained. The resulting apparent $V$ magnitudes and different colors are shown in Table 2. Values found in March 2007 are published in DeMeo et al. (2009).
Table 2. Photometry results.

\begin{tabular}{cccc}
\hline \hline & $V$ & $V-R$ & $V-I$ \\
\hline 20 Mar. 07 & $18.49 \pm 0.05$ & $0.45 \pm 0.07$ & - \\
03 Feb. 08 & $18.79 \pm 0.02$ & $0.45 \pm 0.03$ & $0.91 \pm 0.04$ \\
\hline & $V-J$ & $V-H$ & $V-K$ \\
\hline 20 Mar. 07 & $1.49 \pm 0.07$ & $1.98 \pm 0.08$ & $2.19 \pm 0.08$ \\
03 Feb. 08 & $1.68 \pm 0.06$ & $2.18 \pm 0.050$ & $2.41 \pm 0.06$ \\
\hline
\end{tabular}

\subsection{Spectroscopic data}

Visible spectroscopy was obtained with the low resolution grism (150 grooves per mm) on FORS1 (Mar. 2007 run) and FORS2 (Feb. 2008 run), with a 1" wide slit covering the $0.4-0.94 \mu \mathrm{m}$ range. The resulting spectral resolution is about 200. The slit was oriented along the parallactic angle, to minimize the effects of atmospheric differential refraction, which is also instrumentally corrected at the telescope level. A sorting filter (OG435) was used to eliminate the second order contribution for the first run in service mode (Mar. 2007). For both runs, data reduction was performed using standard Midas and IRAF procedures (see Fornasier et al. 2004). We computed the slope of the resulting spectra using a standard least squares fitting of a linear function, in the wavelength range between 0.5 and $0.8 \mu \mathrm{m}$. The computed visual slopes are: $7.8 \pm 0.5 \% / 0.1 \mu \mathrm{m}$ in March 2007, and $10.4 \pm 0.5 \% / 0.1 \mu \mathrm{m}$ in February 2008. Error bars account for the $1 \sigma$ uncertainty in the linear fit plus $0.5 \% / 0.1 \mu \mathrm{m}$, which we attribute to the use of different analog stars and detector efficiency. Both spectra show an overall concave shape, whereas Barucci et al. (1999) published a spectrum with a convex shape (see Fig. 1, where each spectrum has been normalized at 0.55 microns, and shifted by 0.5 on the plot for clarity). The spectral range covered by the spectrum from Barucci et al. (1999) is different since it was taken at the NTT with a different instrument. For the data presented in this paper, we divided the spectra by different solar-analog star spectra (close in time and airmass), which confirmed Chariklo's visible spectral shape.

We obtained $J$-band spectroscopy with the ISAAC instrument mounted on UT1, using the Short Wavelength Low Resolution grating (resolution of 500) and a $1^{\prime \prime}$ wide slit. We used the ISAAC-dedicated ESO package ECLIPSE to reduce the spectra. We first corrected all data (science and calibrations) for the electrical ghost effect. The master flat, master dark, badpixel map, and distortion correction were created and applied to the data. The wavelength calibration was performed using arc images from Xenon and Argon lamps. For each cube of data, $A B$ pairs were subtracted (A-B and B-A) to correct for the sky contribution and realigned in a single $2 \mathrm{D}$ spectrum image using the ECLIPSE task "sp_jitter". The final 1D spectrum was extracted using the IRAF APEXTRACT/APALL task. We divided the spectra of Chariklo by a solar-analog spectrum observed as close as possible in time and airmass to correct for the telluric lines and remove the contribution from the Sun's spectrum. However, the $J$-band spectrum taken in March 2007 shows some bad sky-line residuals and an increase in the flux at the edges of the spectrum. This is due to the very different airmasses of the object and the solar-analog observations.

We used SINFONI (Spectrograph for INtegral Field Observations in the Near Infrared), mounted on ESO-VLT UT4, to obtain the $H+K$ spectra. SINFONI is equipped with a $2048 \times$ 2048 pixels Rockwell Hawaii 2RD detector. Observations were collected using the $8^{\prime \prime} \times 8^{\prime \prime}$ field of view in seeing-limited conditions. We used the $H+K$ grism, leading to a spectral resolution of about 1500 in the $H$ band. The exposures were centered in time 


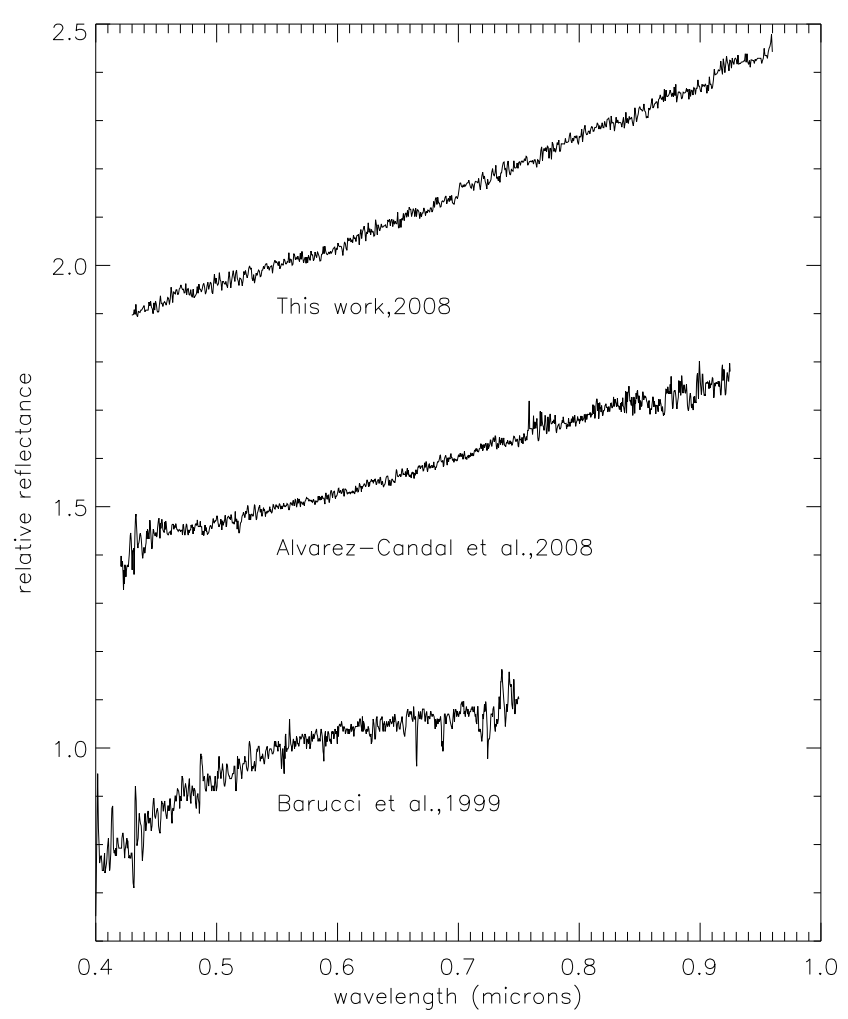

Fig. 1. Chariklo's visible spectrum at different moments.

on Chariklo's meridian transit to ensure stable airmass. Data reduction was performed with SINFONI pipeline 1.7 developed by ESO, and Chariklo's spectra were extracted using QFitsView, developed by MPE (Max-Plack-Institut für Extraterrestrische Physik). Guilbert et al. (2009) provided further details of the data reduction. Chariklo's spectrum was finally divided by a solar analog spectrum to correct for the telluric lines and the solar response. Observational conditions are summarized in Table 3: for each instrument used, we present the different filters used during the two observing runs, the exposure time, the airmass, and the solar analog as long as its airmass. FORS data taken in 2007 are published in Alvarez-Candal et al. (2008), SINFONI data taken in 2007 are published in Guilbert et al. (2009).

\subsection{Search for absorption bands}

\subsubsection{Visible spectrum}

To search for the presence of absorption bands in the visible spectrum, we followed the method described in Vilas et al. (1993). We computed a linear fit to the spectra in the $0.5-0.8 \mu \mathrm{m}$ wavelength range, and then removed it from the spectra as a form of continuum. We found that both spectra exhibit a shallow, visible in Fig. 2, which shows Chariklo's spectra divided by a linear continuum.

The visible spectrum obtained in March 2007 is very similar to the spectrum of $2003 \mathrm{AZ}_{84}$ (Alvarez-Candal et al. 2008). They both exhibit a $0.3 \mu \mathrm{m}$ wide shallow absorption band, centered on about $0.65 \mu \mathrm{m}$. In the case of Chariklo, this band has a depth of $1 \%$. The same feature is detected in Chariklo's spectrum observed in February 2008, with a depth of $2 \%$. The two spectra were corrected for the solar response and telluric features by using two different solar-analog stars. These stars were used to correct the spectra of other objects, for which we did not detect any feature in the visible part of the spectrum. We therefore believe that this absorption band is real in both of Chariklo's spectra, and that it could be attributed to the presence of hydrated minerals on Chariklo's surface. The $0.65 \mu \mathrm{m}$ feature indeed resembles absorption bands that were detected in some dark primitive asteroid spectra, attributed to the presence on the surface of minerals produced by the aqueous alteration of anhydrous silicates (Vilas \& Gaffey 1989; Vilas et al. 1994). This band is produced by a $\mathrm{Fe}^{2+} \longrightarrow \mathrm{Fe}^{3+}$ charge transfer transition in phyllosilicates. Nonetheless, this absorption band is detected around $0.7 \mu \mathrm{m}$ in the case of asteroids.

\subsubsection{Near-Infrared spectrum}

Though the $H+K$ spectra look featureless, we attempted to compute the depth of a potential absorption band produced by water ice around $2.0 \mu \mathrm{m}$, to place constraints on the amount of water ice present in these spectra. We therefore applied an algorithm that computes first the median of the reflectance in a 11-pixel box, centered on about 1.8, 2.0, and $2.2 \mu \mathrm{m}$. A linear continuum between the median reflectance around $1.8 \mu \mathrm{m}$ and $2.2 \mu \mathrm{m}$ was then determined. The depth of the band was calculated relative to the continuum reflectance around $2.0 \mu \mathrm{m}$. The $K$-band spectrum taken in March 2007 shows a telluric feature at $2.03 \mu \mathrm{m}$, which is not properly corrected by the solar analog. To compute the depth of a potential absorption band in this spectrum, we replaced the region of the telluric feature by an interpolation of the spectrum. We measured a depth of $3 \pm 2 \%$ (see Table 5). For the spectrum taken in February 2008, the depth of this potential absorption band is $2 \pm 5 \%$. Therefore, we cannot exclude the possible presence of a $2.0 \mu \mathrm{m}$ absorption band due to water ice in these two spectra. No other feature can be detected within the uncertainties created by the noise in both NIR spectra.

Phyllosilicates also have absorption bands at 1.4, 1.9, and 2.2-2.4 $\mu \mathrm{m}$ (Hunt 1977). If we believe that phyllosilicates are responsible for the potential $0.65 \mu \mathrm{m}$ band detected on both Chariklo's visible spectra, we should detect these other bands. The 1.4 and $1.9 \mu \mathrm{m}$ bands appear in the region of atmospheric cut-off., and we were therefore unable to detect these two features. No 2.2-2.4 $\mu \mathrm{m}$ absorption band can be detected within the uncertainties created by the noise in both Chariklo's K spectra. In conclusion, we believe that the feature detected in the visible spectrum could be caused by some aqueous alteration product, but we are unable to confirm the presence of phyllosilicates.

\section{Data analysis by spectral modeling}

\subsection{Surface components}

To model Chariklo's spectra, we considered compounds that could be present on its surface. The components are chosen a priori according to both our knowledge of Centaurs and the absorption features found in Chariklo's spectra. We are facing a low albedo surface ( $p_{\mathrm{v}}=5.7 \%$, from Stansberry et al. 2008), slightly red, and without any strong absorption features, although aqueous alterated minerals could be present (absorption bands in the visible spectra).

Ices. Water ice bands were identified in Chariklo's NIR spectrum (Brown et al. 1998; Brown \& Koresko 1998; Dotto et al. 2003), so water should be and was included in the model. Other ices might be present on Chariklo's surface, but no clear NIR absorption features were identified in its spectrum. This would suggest that their abundance on the surface is well below 10\% (see discussion on minor components in Pinilla-Alonso et al. 2009), so we did not introduce any other volatile compounds in our 
Table 3. Observational conditions for spectroscopic observations.

\begin{tabular}{lccccc}
\hline \hline & Run & Filter & $\begin{array}{c}\text { Exp. time } \\
(\mathrm{sec})\end{array}$ & Airmass & $\begin{array}{c}\text { Analog } \\
\text { (airmass) }\end{array}$ \\
\hline \multirow{2}{*}{ FORS } & 20 Mar. 07 & Vis & 2000 & 1.02 & SA 107 998(1.22) \\
& 03 Feb. 08 & Vis & 900 & 1.1 & Ld 102 1081(1.13) \\
ISAAC & 20 Mar. 07 & $J$ & 1200 & 1.14 & SA 107 998(1.83) \\
& 03 Feb. 08 & $J$ & 1080 & 1.09 & Hip 045 953(1.06) \\
\multirow{2}{*}{ SINFONI } & 20 Mar. 07 & $H+K$ & 4800 & 1.04 & SA 107 998 (1.21) \\
& 03 Feb. 08 & $H+K$ & 4200 & 1.07 & Hip 071 136(1.04) \\
\hline
\end{tabular}

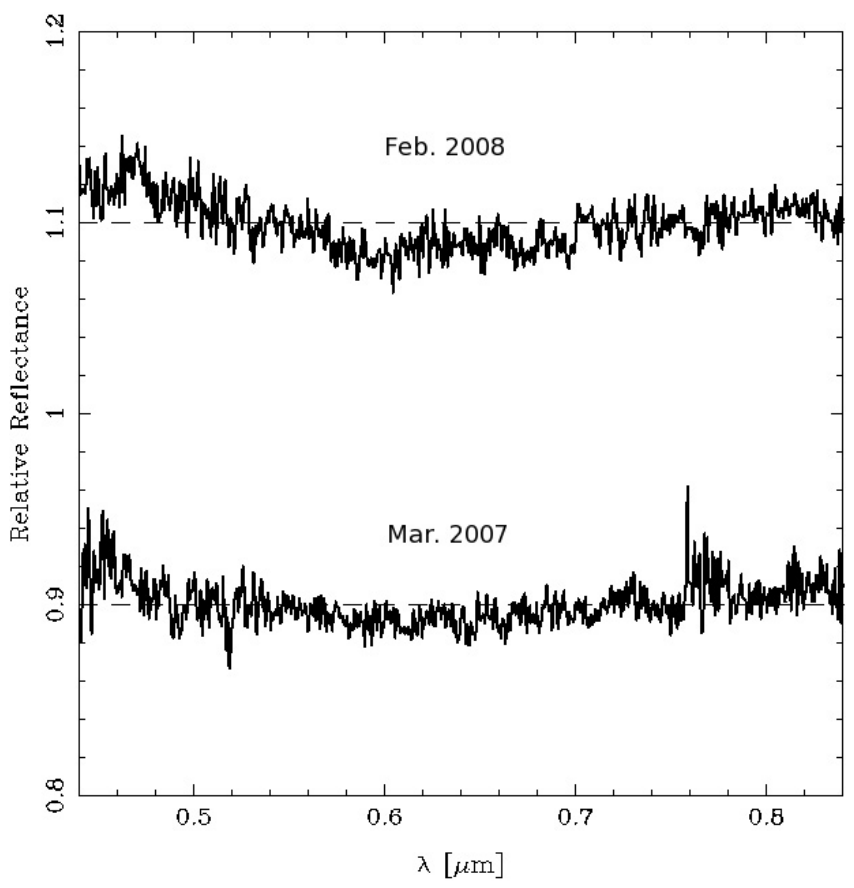

Fig. 2. Chariklo's visible spectra divided by a linear continuum to search for subtle bands.

modeling. We added both crystalline and amorphous water ice (optical constants from Grundy \& Schmitt 1998 for crystalline water ice at $40 \mathrm{~K}$, and from Schmitt et al. 1998 for amorphous water ice at $38 \mathrm{~K})$.

Tholins. Because of a lack of adequate optical constants, tholins (Khare et al. 1984) are commonly used in minor body spectral models, to represent a very broad class of complex organic solids (Cruikshank et al. 2005). They are complex polymer-like compounds (Mutsukura \& Akita 1999; Bernard et al. 2006), which might be present on Trans-Neptunian objects' and Centaurs' surfaces (Cruikshank et al. 1998). In the laboratory, tholins are produced by energetic processing of $\mathrm{N}_{2}: \mathrm{CH}_{4}$ gaseous mixtures. Triton tholins $\left(99.9 \% \mathrm{~N}_{2}\right.$ and $0.01 \% \mathrm{CH}_{4}$, McDonald et al. 1994) and Titan tholins $\left(90 \% \mathrm{~N}_{2}\right.$ and $10 \% \mathrm{CH}_{4}$, Khare et al. 1984) were included.

Amorphous carbon. We know that space weathering processes on C-bearing ices, such as $\mathrm{CH}_{4}$ or $\mathrm{CH}_{3} \mathrm{OH}$, produce organic residues (Andronico et al. 1987). A prolonged irradiation of these organics can result in the production of a spectrally dark and featureless amorphous carbon. We therefore added some amorphous carbon in our spectral modeling, to reproduce the surface's low albedo (optical constants from Zubko et al. 1996).

Silicates. Silicates are thought to be ubiquitously present on minor bodies, but they are not easily detectable when mixed with high amounts of carbons. No phyllosilicates were added since no optical constants were available for these compounds. Nonetheless, we included olivine as a Fe-bearing component.

\subsection{Models}

Our spectral modeling was based on the model developed by Shkuratov et al. (1999). It is applied to the entire visible+nearinfrared wavelength range, to obtain the fit that reproduces the spectrum most accurately (Merlin et al. 2007). This best fit corresponds to the composition and grains sizes minimizing the value of the reduced $\chi^{2}$. Visible spectra were normalized at $0.55 \mu \mathrm{m}$ to $p_{\mathrm{v}}=0.057$ (Stansberry et al. 2008). The different parts of the spectra were adjusted using the photometric colors published in DeMeo et al. (2009) and in this work.

Model 1. We first used an intimate mixture of all compounds mentioned in Sect. 3.1. The spectrum taken in March 2007 were modeled by a mixture of $3 \%$ of crystalline water ice $(10 \mu \mathrm{m}$ grains), $5 \%$ of amorphous water ice (10 $\mu$ m grains), $6 \%$ of Triton tholins (5 $\mu \mathrm{m}$ grains) and $86 \%$ of amorphous carbon $(10 \mu \mathrm{m}$ grains). The second spectrum's (Feb. 2008) best fit includes 5\% of crystalline water ice (18 $\mu \mathrm{m}$ grains), $4 \%$ of amorphous water ice (10 $\mu \mathrm{m}$ grains), $17 \%$ of Triton tholins (16 $\mu \mathrm{m}$ grains), and $74 \%$ of amorphous carbon (10 $\mu \mathrm{m}$ grains). In both cases, the fractions of Titan tholins and olivine were found to be less than 1\%o. Results are presented in Fig. 3: dashed lines correspond to Chariklo's extracted spectra obtained in March 2007 (bottom) and February 2008 (top). The spectrum taken in February 2008 has been shifted by 0.02 in the plot for clarity. The solid lines correspond to the best-fit models.

Model 2. In a second time, we used a geographical mixture of all compounds mentioned in Sect. 3.1. These would thus be distributed as different patches on the surface, which would agree with the suspected heterogeneity. Chariklo's spectrum taken in March 2007 was modeled with a geographical mixture of $95 \%$ amorphous carbon (10 $\mu \mathrm{m}$ grains), $2 \%$ Triton and Titan tholins (40 and $50 \mu \mathrm{m}$ grains respectively), and $1 \%$ olivine (10 $\mu \mathrm{m}$ grains). The spectrum taken in February 2008 consists of $87 \%$ amorphous carbon (10 $\mu \mathrm{m}$ grains), $6 \%$ Triton and Titan tholins (70 and $15 \mu \mathrm{m}$ grains respectively), and $1 \%$ olivine (10 $\mu \mathrm{m}$ grains). Results are presented in Fig. 4: dashed lines correspond to Chariklo's extracted spectra obtained in March 2007 (bottom) and February 2008 (top). The spectrum taken in February 2008 has been shifted by 0.02 in the plot for clarity.

These results illustrate that the composition found by spectral modeling is only indicative and not unique. A composition of up to $10 \%$ of water ice could be present in both spectra using intimate mixtures, while no water ice is included in the geographical mixtures. Amorphous carbon is the major component $(>80 \%)$ in both spectra, using both types of mixtures. 


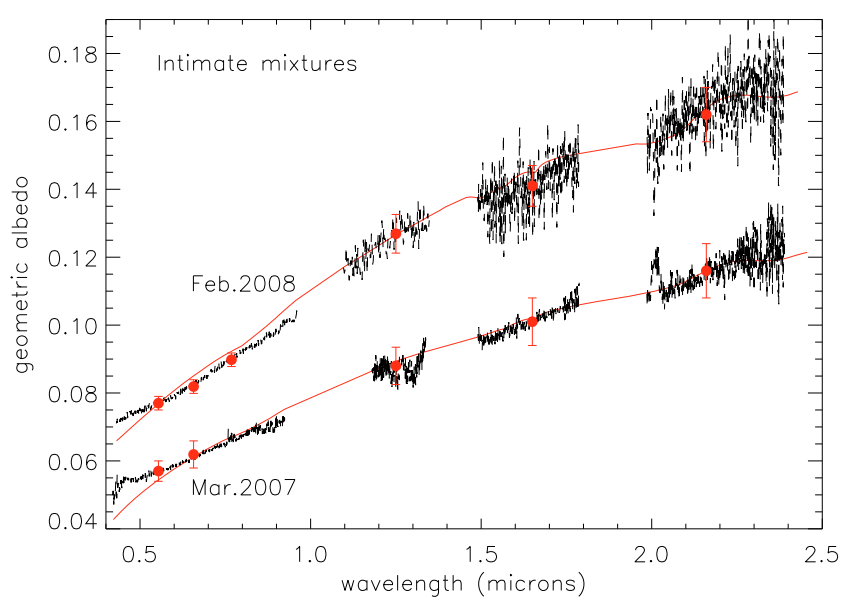

Fig. 3. Results of the spectral modeling using intimate mixtures.

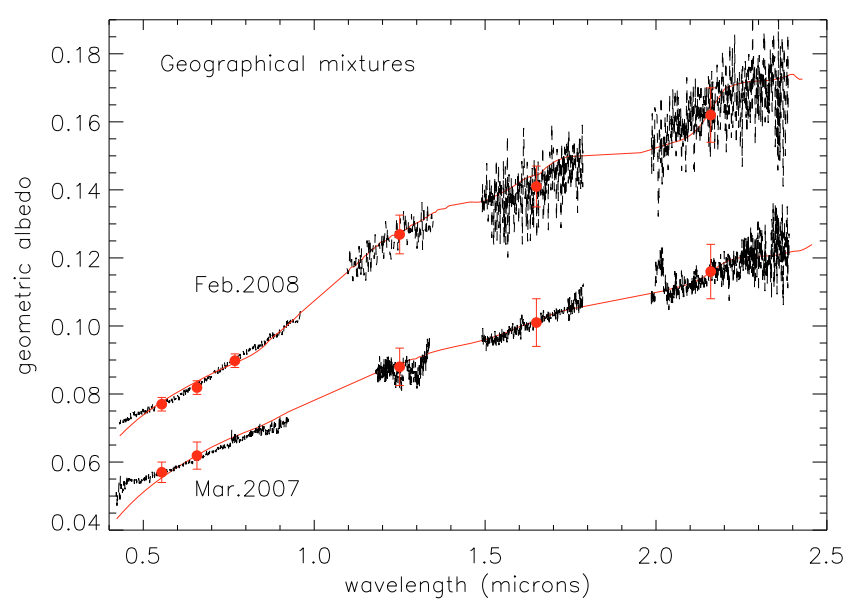

Fig. 4. Results of the spectral modeling using geographical mixtures.

\subsection{Comparison with previous works}

Chariklo's visible spectrum exhibits variations in its spectral behaviour. The spectrum taken by Barucci et al. (1999) in 1998 has indeed a convex shape, without any absorption band, whereas the spectra obtained in the framework of the ESO Large Program (Alvarez-Candal et al. 2008, and the new spectrum presented in this work) are concave and show a wide feature around $0.65 \mu \mathrm{m}$ (see Fig. 1), which could be attributed to minerals produced by aqueous alteration. Some variation in the spectral slope is also observed between the spectra taken in March 2007 and February 2008: from $7.8 \pm 0.5 \% / 0.1 \mu \mathrm{m}$ (Alvarez-Candal et al. 2008) to $10.4 \pm 0.5 \% / 0.1 \mu \mathrm{m}$ in this work. In the NIR spectra, the spectral behaviour variations can be clearly noticed, as shown in Fig. 5 . For all previous data, absorption bands at 1.5 and $2.0 \mu \mathrm{m}$ of varying depths are detected, indicating the presence of water ice (Brown et al. 1998; Brown \& Koresko 1998; Dotto et al. 2003). Our two spectra one from (Guilbert et al. 2009 and the new spectrum presented in this work) show a different behaviour, with no features. These results confirm that Chariklo's surface might contain some inhomogeneities.

Table 4 summarizes all modeling results found for Chariklo: year of observation, spectral model used, wavelength range over which it is applied, type of mixture, visual albedo, water ice amount found by the best-fit model, and the amount of other components. However, we note that this compilation of spectral models highlights the difficulty of interpreting the surface

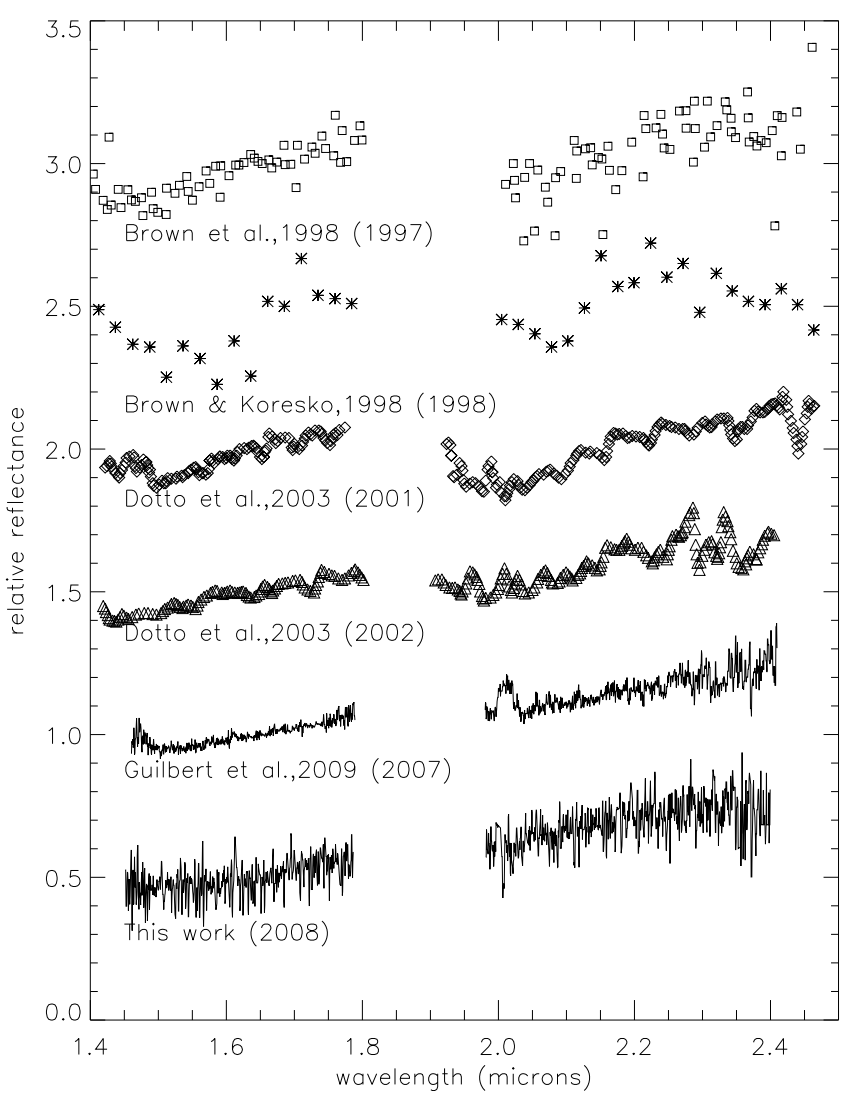

Fig. 5. $H+K$ spectrum of Chariklo obtained by different groups. The observing date is indicated into brackets.

composition. From our data set, water ice may be present on Chariklo's surface with an abundance ranging from zero to a few percent. The bands depth seems more reliable when comparing the different spectra. We computed the depths of the $2.0 \mu \mathrm{m}$ band of all spectra using the same technique described in Sect. 3.2. The results can be found in Table 5 .

\section{Discussion}

\subsection{Aqueous alteration}

Chariklo's spectrum might present evidence of hydrated minerals, because of the detection of a small, shallow, absorption band around $0.65 \mu \mathrm{m}$. This band is similar to a feature detected in the spectra of dark asteroids, which was attributed to the presence of minerals produced by the aqueous alteration of anhydrous silicates (Vilas \& Gaffey 1989; Vilas et al. 1994). Minerals produced by aqueous alteration are detected not only on dark asteroids, but also on carboneous meteorites, interplanetary dust and micrometeorites, as well as on some comet nuclei (Lisse et al. 2006).

Hydrated minerals might have formed in the early phases of Chariklo's chemical evolution. Aqueous alteration indeed occurs by means of two main mechanism: either low temperature alteration (temperature between 273 and $400 \mathrm{~K}$ ), or hydrocryogenic alteration (temperature between 200 and $273 \mathrm{~K}$ ) (Rietmeijer 1985; Rietmeijer \& Mackinnon 1987). When it comes to Chariklo, two heat sources might be considered: the transient heat generated by impacts, and the heat released by radiogenic decay. Although the melting of water ice could be common inside icy bodies (McKinnon et al. 2008), it is more probable for the largest objects, since it requires not only 
Table 4. Comparison of different models.

\begin{tabular}{|c|c|c|c|c|c|c|c|}
\hline Reference & Date & Model & $\lambda$ range & Mix. & $p_{v}$ & $\mathrm{H}_{2} \mathrm{O}$ amount & Other components \\
\hline Br. \& Ko. & 1998 & Hapke & $H+K$ & Geo. & 0.050 & $3 \%(10 \mu \mathrm{m})$ & $\begin{array}{l}\text { Murchison 97\% } \\
\text { Am. C } 80 \%(10 \mu \mathrm{m})\end{array}$ \\
\hline Dotto et al. & 2001 & Hapke & Vis $+J+H+K$ & Geo. & 0.059 & $2 \%(20 \mu \mathrm{m})$ & $\begin{array}{l}\text { Triton th. } 13 \%(10 \mu \mathrm{m}) \\
\text { Titan th. } 5 \%(7 \mu \mathrm{m})\end{array}$ \\
\hline Dotto et al. & 2002 & Hapke & Vis $+J+H+K$ & Geo. & 0.055 & $2 \%(20 \mu \mathrm{m})$ & $\begin{array}{l}\text { Am. C } 84 \%(10 \mu \mathrm{m}) \\
\text { Triton th. } 7 \%(10 \mu \mathrm{m}) \\
\text { Titan th. } 7 \%(7 \mu \mathrm{m})\end{array}$ \\
\hline This work & 2007 & Shkur. & $\begin{array}{l}\text { Simultaneous } \\
\text { Vis }+J+H+K\end{array}$ & Geo. & 0.057 & No water ice & $\begin{array}{l}\text { Am. C } 95 \%(10 \mu \mathrm{m}) \\
\text { Triton th. } 2 \%(40 \mu \mathrm{m}) \\
\text { Titan th. } 2 \%(50 \mu \mathrm{m}) \\
\text { Olivine } 1 \%(10 \mu \mathrm{m})\end{array}$ \\
\hline This work & 2008 & Shkur. & $\begin{array}{l}\text { Simultaneous } \\
\text { Vis }+J+H+K\end{array}$ & Geo. & 0.057 & No water ice & $\begin{array}{l}\text { Am. C } 87 \%(10 \mu \mathrm{m}) \\
\text { Triton th. } 6 \%(70 \mu \mathrm{m}) \\
\text { Titan th. } 6 \%(15 \mu \mathrm{m}) \\
\text { Olivine } 1 \%(10 \mu \mathrm{m})\end{array}$ \\
\hline This work & 2007 & Shkur. & $\begin{array}{l}\text { Simultaneous } \\
\text { Vis }+J+H+K\end{array}$ & Int. & 0.057 & $\begin{array}{l}\text { Cr. 3\% }(10 \mu \mathrm{m}) \\
\text { Am. } 5 \%(10 \mu \mathrm{m})\end{array}$ & $\begin{array}{l}\text { Am. C } 86 \%(10 \mu \mathrm{m}) \\
\text { Triton th. } 6 \%(5 \mu \mathrm{m})\end{array}$ \\
\hline This work & 2008 & Shkur. & $\begin{array}{l}\text { Simultaneous } \\
\text { Vis }+J+H+K\end{array}$ & Int. & 0.057 & $\begin{array}{l}\text { Cr. } 5 \%(18 \mu \mathrm{m}) \\
\text { Am. } 4 \%(10 \mu \mathrm{m})\end{array}$ & $\begin{array}{l}\text { Am. C } 74 \%(10 \mu \mathrm{m}) \\
\text { Triton th. } 17 \%(16 \mu \mathrm{m})\end{array}$ \\
\hline
\end{tabular}

Table 5. Depth of the $2.0 \mu \mathrm{m}$ absorption band due to water ice in Chariklo's spectrum taken at different periods.

\begin{tabular}{lccc}
\hline \hline Reference & Date & $d_{H}$ (AU) & Depth \\
\hline Brown et al. (1998) & 27 Oct. 1997 & 13.799 & $20 \pm 6 \%$ \\
Brown \& Koresko (1998) & 07 Mar. 1998 & 13.725 & $20 \pm 8 \%$ \\
Dotto et al. (2003) & 17 Apr. 2001 & 13.235 & $17 \pm 4 \%$ \\
Dotto et al. (2003) & 10 Mar. 2002 & 13.154 & $7 \pm 4 \%$ \\
Guilbert et al. (2009) & 20 Mar. 2007 & 13.277 & $3 \pm 2 \%$ \\
This work & 03 Feb. 2008 & 13.395 & $0 \pm 3 \%$ \\
\hline
\end{tabular}

$d_{H}$ : heliocentric distance for the different observing runs.

high temperatures $(>273 \mathrm{~K})$ but also high pressures $(>600 \mathrm{~Pa})$ (Prialnik et al. 2008). We therefore need to study whether radiogenic elements have been sufficient to heat the body in the early phases of its life, and if the heat flow could have extended to the surface, enabling the hydrated minerals to reach the surface.

Aqueous alteration might also occur on the surface upon irradiation by energetic particles. Although a detailed study of space weathering effects in conjunction with aqueous alteration has not yet been performed, some hints can be obtained by irradiation experiments performed on samples containing phyllosilicates (OH-rich). Brunetto \& Strazzulla (2005) reported reflectance spectra of irradiated silicates rich in serpentine, but no clear trend was found between the weak features at about 1.38, 1.95 , and $2.32 \mu \mathrm{m}$ (attributed to $\mathrm{OH}$ vibrations) and the irradiation dose. At the present time, we cannot exclude the possibility that irradiation of silicates mixed with water ice can induce additional hydration. Such a process has been found to be efficient for fused silicate glasses (Arnold et al. 2000).

\subsection{Irradiation}

Strazzulla et al. (1991) and Brunetto \& Roush (2008) demonstrated that if a surface is heavily irradiated, a crust may be produced that can easily mask the presence of volatiles. From the results presented in Sect. 3., we can assume that Chariklo's upper layer should consist of less than $20 \%$ water ice and organics, and a large amount $(>80 \%)$ of highly processed amorphous carbon, maybe produced by irradiation.

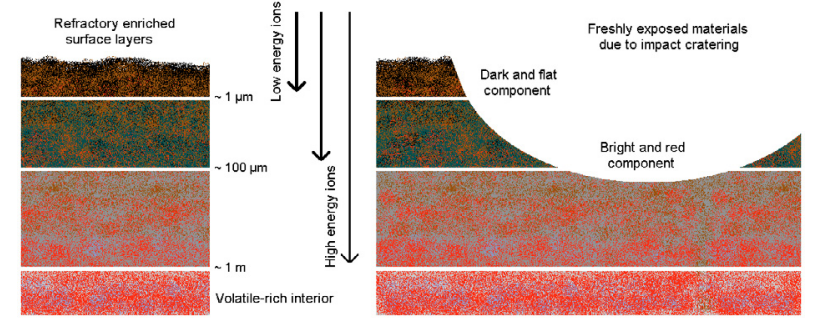

Fig. 6. Illustration of particle irradiation by different energies.

Hudson et al. (2008) reported estimates of the radiation doses for objects at different distances from the Sun. On a timescale of about $4.6 \times 10^{9}$ years, Centaurs orbiting at 5-35 AU (which is the case for Chariklo) accumulate an irradiation dose of about 100$10000 \mathrm{eV} / 16$-amu, 100-200 eV/16-amu, and $30 \mathrm{eV} / 16$-amu, in the outer $1 \mu \mathrm{m}, 100 \mu \mathrm{m}$, and 1 meter deep layer, respectively (see Fig. 6). Assuming a lifetime of 10 Myr (Tiscareno \& Malhotra 2003) and considering that Chariklo's semi-major axis is about $16 \mathrm{AU}$ (its present orbit is confined to between about $13 \mathrm{AU}$ and 19 AU), using Hudson et al. (2008) results, we can reasonably estimate a dose of about $2 \mathrm{eV} / 16$-amu received by the first micrometers of Chariklo's surface during its "centaur phase". This dose would be able to induce some destruction of simple C-bearing molecules (Brunetto et al. 2006), but would not be enough to produce a layer of highly refractory de-hydrogenated carbons (Brunetto \& Roush 2008).

Hence, if Chariklo's amorphous carbon is a by-product of a prolonged space weathering effect, we should conclude that this component formed in a different environment than its current one, i.e., in the trans-neptunian region if Centaurs are formed there. This also depends on the formation region of Chariklo. As shown by Hudson et al. (2008), in the present outer solar system conditions TNOs orbiting between 40 and $48 \mathrm{AU}$ indeed receive much lower doses (about a factor of 100-1000) than those orbiting beyond the termination shock (about $85 \mathrm{AU}$ ). These doses would not be enough to explain the amount of amorphous carbon observed on Chariklo, and one should conclude that the refractory carbonaceous component is primordial. In contrast, objects orbiting beyond the termination shock can accumulate high doses (>200 eV/16-amu) and their upper layers can be deeply 
Table 6. Visible and near-infrared colors of Chariklo, determined by different groups.

\begin{tabular}{cccccc}
\hline \hline$V-R$ & $V-I$ & $V-J$ & $V-H$ & $V-K$ & Ref. \\
\hline $0.46 \pm 0.02$ & $1.01 \pm 0.02$ & $1.67 \pm 0.10$ & $2.08 \pm 0.10$ & $2.17 \pm 0.10$ & Davies et al. (1998) \\
$0.46 \pm 0.02$ & $1.02 \pm 0.02$ & $1.74 \pm 0.02$ & $2.15 \pm 0.02$ & $2.25 \pm 0.02$ & McBride et al. (1999) \\
$0.45 \pm 0.07$ & - & $1.49 \pm 0.07$ & $1.98 \pm 0.08$ & $2.19 \pm 0.08$ & DeMeo et al. (2009) \\
$0.45 \pm 0.03$ & $0.91 \pm 0.04$ & $1.68 \pm 0.06$ & $2.18 \pm 0.05$ & $2.41 \pm 0.06$ & This work, 2008 \\
\hline
\end{tabular}

affected by irradiation-induced chemistry, and eventually form amorphous carbon crusts (Brunetto \& Roush 2008). This would therefore suggest a link between Chariklo and scattered disk objects, which is also supported by dynamical studies (Gomes et al. 2008, and references therein).

\subsection{Comet-like activity}

The variations in the different spectra of Chariklo might be caused by temporal variations, as in the case of Chiron. Luu et al. (2000) showed that the detection of water ice features on Chiron's spectrum is time-variable, and anti-correlated with the level of comet-like activity. These features are detected in Chiron's spectra with variable depths related to a variable dilution of the nucleus' signal by the coma. This could hence be the case for Chariklo, which is very close to its perihelion during the different observing runs ( $q=13.084 \mathrm{AU}$, reached in 2004). We therefore checked the possibility of Chariklo exhibiting some comet-like activity with an evolving coma. Although Chariklo's perihelion distance is farther from the Sun than Chiron's one ( $q=8.506 \mathrm{AU}$ in the case of Chiron), cometary activity at large heliocentric distances has already been observed (Meech \& Belton 1990; Rousselot 2008). Photometric colors are consistent with the previously published sets of colors (see Table 6). Our images do not show the presence of any obvious coma, and the point spread functions of Chariklo and stars in the field are the same within the error bars, as confirmed by the inspection of all visible images available within our LP dataset. We therefore conclude that we are unable to detect any significant comet-like activity in our data, although we cannot discard the presence of a coma within uncertainty levels due to the noise.

Furthermore, Merlin et al. (2005) showed that in the case of Thereus, the differences in the spectra observed could be due to surface variations rather than temporal variations. Both types of variations are not in disagreement with the observed variations on Chariklo. Surface variations are investigated in the next section.

\subsection{Variation of the composition}

As suggested by Strazzulla et al. (1991) and Brunetto \& Roush (2008), a highly processed thin crust can hide layers underneath, also affected by irradiation, in which organics are formed and ices can survive, as illustrated in Fig. 7. The underlying material can therefore be exposed to the surface by impact cratering (see Fig. 6). Chariklo's spectrum can be reproduced by considering the contribution of these two different materials: the highly processed one, and the underlying less processed one. This is illustrated in Fig. 7, which shows three synthetic spectra.

The Chariklo-like mixture is obtained from our spectral modeling, by fitting of the spectrum obtained in March 2007. The dark and flat spectrum should correspond to a totally processed carboneous surface. It is produced by removing all water ice and organics from the Chariklo-like mixture, and retaining the proportions of irradiated materials such as amorphous carbon. The bright and red spectrum is understood to reproduce

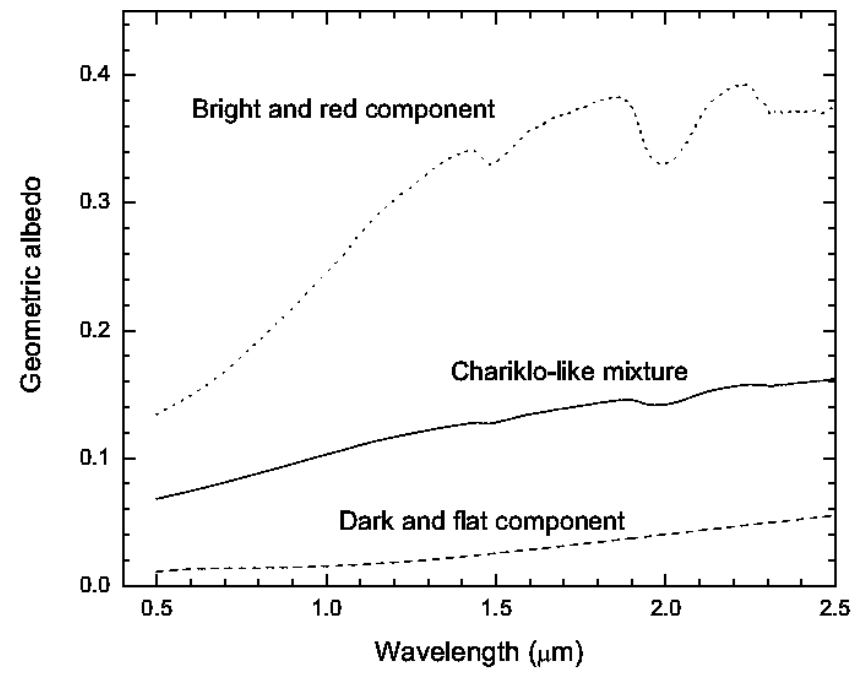

Fig. 7. Interpretation of Chariklo's spectral behaviour in terms of space weathering processes and impacts.

the composition of a given underlying layer, affected by irradiation with lower doses. We indeed reproduced the composition by maintaining the proportion of water ice and organics found in the Chariklo-like mixture, and reducing the amount of irradiated material. We could reproduce the variations observed in Chariklo's spectral behaviour by mixing those two spectra by different amounts. We emphasize that this description is not meant to be a definite compositional interpretation, we wish only to provide a qualitative interpretation of the observed variations.

Hence, the observation of water ice on Chariklo is not in conflict with the presence of irradiation-produced amorphous carbon. We can assume that part of the surface has been resurfaced by impacts (Fig. 6). The varied cratering activity affecting the surface may expose different amounts of fresh material, thus leading to the different spectral behaviours presented in this work.

Acknowledgements. We acknowledge A. Doressoundiram for his usefull comments on the discussion. R.B. acknowledges support from the French "Agence Nationale de la Recherche" (contract ANR-05-BLAN-0148-02).

\section{References}

Altenhoff, W. J., Menten, K. M., \& Bertoli, F. 2001, A\&A, 366, 9

Alvarez-Candal, A., Fornasier, S., Barucci, et al. 2008, A\&A, 487, 741

Andronico, G., Baratta, G. A., Spinella, F., et al. 1987, A\&A, 184, 333

Arnold, G. W., Battaglin, G., Mattei, G., et al. 2000, Nucl. Instr. Methods Phys. Res. B, 166, 440

Barucci, M. A., Lazzarin, M., \& Tozzi, G. P. 1999, AJ, 117, 1929

Barucci, M. A., Brown, M. E., Emery, J. P., et al. 2008, in The Solar System Beyond Neptune, ed. M. A. Barucci, H. Boehnhardt, D. Cruikshank, \& A. Morbidelli, 143

Bernard, J.-M., Quirico, E., Brissaud, O., et al. 2006, Icarus, 185, 301

Brown, R. H., Cruikshank, D. P., Pendleton, Y., et al. 1998, Sci., 280, 1430

Brown, M. E., \& Koresko, C. C. 1998, ApJ, 505, 65

Brunetto, R., \& Roush, T. L. 2008, A\&A, 481, 879

Brunetto, R., \& Strazzulla, G. 2005, Icarus, 179, 265

Brunetto, R., Barucci, M. A., Dotto, E., et al. 2006, ApJ, 644, 646 
Cruikshank, D. P., Roush, T. L., Bartholomew, M. J., et al. 1998, Icarus, 135, 389 Cruikshank, D. P., Imanaka, H., \& Dalle Ore, C. M. 2005, Adv. Space Res., 36, 178

Davies, J. K., McBride, N., Ellison, et al. 1998, Icarus, 134, 213

DeMeo, F., Fornasier, S., Barucci, M. A., et al. 2009, 493, 283

Dotto, E., Barucci, M. A., Leyrat, C., et al. 2003, Icarus, 164, 122

Fornasier, S., Doressoundiram, A., Tozzi, G. P., et al. 2004, A\&A, 421, 353

Fulchignoni, M., Belskaya, I., Barucci, M. A., et al. 2008, in The Solar System Beyond Neptune, ed. M. A. Barucci, H. Boehnhardt, D. Cruikshank, \& A. Morbidelli, 181

Gomes, R. S., Fernández, J. A., Gallardo, T., et al. 2008, in The Solar System Beyond Neptune, ed. M. A. Barucci, H. Boehnhardt, D. Cruikshank, \& A. Morbidelli, 259

Groussin, O., Lamy, P., \& Jorda, L. 2004, A\&A, 413, 1163

Grundy, W. M., \& Schmitt, B. 1998, J. Geophys. Res., 103, 25809

Guilbert, A., Alvarez-Candal, A., Merlin, F., et al. 2009, Icarus, 201, 272

Hudson, R. L., Palumbo, M. E., Strazzulla, et al. 2008, The Solar System Beyond Neptune, 507

Hunt, G. R. 1977, Geophysics, 42, 501

Hunt, G. R., \& Ashley, R. P. 1979, Econ. Geol., 74, 1613

Jewitt, D., \& Kalas, P. 1998, ApJ, 499, 103

Jones, T. D., Lebofsky, L., Lewis, J., et al. 1990, Icarus, 88, 172

Khare, B. N., Sagan, C., Arakawa, E. T., et al. 1984, Icarus, 60, 127

Lisse, C. M., \& 16 colleagues 2006, Nature, 313, 635

Luu, J. X., Jewitt, D. C., \& Trujillo, C. 2000, ApJ, 531, 151

McBride, N., Davies, J. K., Green, S. F., et al. 1999, MNRAS, 306, 799

McDonald, G. D., Thompson, W. R., Heinrich M., et al. 1994, Icarus, 108, 137

McKinnon, W. B., Prialnik, D., Stern, S. A., et al. 2008, in The Solar System Beyond Neptune, ed. M. A. Barucci, H. Boehnhardt, D. Cruikshank, \& A. Morbidelli, 213
Meech, K. J., \& Belton, M. J. S. 1990, AJ, 100, 1323

Merlin, F., Barucci, M. A., Dotto, E., et al. 2005, A\&A, 444, 977

Merlin, F., Guilbert, A., Dumas, C., et al. 2007, A\&A, 466, 1185

Mutsukura, N., \& Akita, K.-I. 1999, Thin Solid Films, 349, 115

Peixinho, N., Lacerda, P., Ortiz, J. L., et al. 2001, A\&A, 371, 753

Pinilla-Alonso, N., Brunetto, R., Licandro, J., et al. 2009, A\&A, in press

Prialnik, D., Sarid, G., \& Rosenberg, E. D. 2008, Space Sci. Rev., 138, 147

Rietmeijer, F. J. M. 1985, Nature, 313, 293

Rietmeijer, F. J. M., \& Mackinnon, I. D. R. 1987, in Symposium on the Diversity and Similarity of Comets, ESA SP-278, 363

Rousselot, P. 2008, A\&A, 480, 543

Schmitt, B., Quirico, E., Trotta, F., et al. 1998, Ap\&SS, 227, ed. Schmitt, B., de Bergh, C., \& Festou, M., 199

Scotti, J. V. 1997, Minor Planet Circular 1997 D-11

Shkuratov, Y., Starukhina, L., Hoffmann, H., et al. 1999, Icarus, 137, 235

Stansberry, J., Grundy, W., Brown, M., et al. 2008, in The Solar System Beyond Neptune, ed. M. A. Barucci, H. Boehnhardt, D. Cruikshank, \& A. Morbidelli, 161

Strazzulla, G., Baratta, G. A., Johnson, R. E., et al. 1991, Icarus, 91, 101

Tegler, S. C., Bauer, J. M., Romanishin, W., et al. 2008, in The Solar System Beyond Neptune, ed. M. A. Barucci, H. Boehnhardt, D. Cruikshank, \& A. Morbidelli, 105

Tiscareno, M., \& Malhotra, R. 2003, AJ, 126, 3122

Townsend, T. E. 1987, J. Geophys. Res., 92, 1441

Vilas, F., \& Gaffey, M. J. 1989, Science, 246, 790

Vilas, F., Larson, S. M., Hatch, E. C., et al. 1993, Icarus, 105, 67

Vilas, F., Jarvis, K. S., \& Gaffey, M. J. 1994, Icarus, 109, 274

Zubko, V., Mennella, V., Colangeli, L., et al. 1996, in The Role of Dust in the Formation of Stars, ed. H. U. Käufl \& R. Siebenmorgen, 333 